Communication

\title{
Medical Hypothesis: Deep Brain Stimulation for Intractable Migraine
}

\author{
Parisa Gazerani ${ }^{1,}{ }^{*}$, Brian E Cairns ${ }^{2}$
}

1. Department of Health Science and Technology, Faculty of Medicine, Aalborg University, Denmark; E-Mail: gazerani@hst.aau.dk

2. Faculty of Pharmaceutical Sciences, The University of British Columbia, Vancouver, Canada; EMail: brian.cairns@ubc.ca

* Correspondence: Parisa Gazerani; E-Mail: gazerani@hst.aau.dk

Academic Editor: Lilach Soreq

Special Issue: Deep Brain Stimulation for Neurobiology Diseases

OBM Neurobiology

2021, volume 5, issue 1

doi:10.21926/obm.neurobiol.2101082
Received: October 26, 2020

Accepted: December 29, 2020

Published: January 05, 2021

\begin{abstract}
Deep Brain Stimulation (DBS) has been introduced as an innovative treatment for several neurological disorders, including Parkinson's disease, essential tremor, and dystonia and it has been proven effective in helping individuals affected by these disorders; hence, enhancing their quality of life. DBS has also been investigated for the treatment of cluster and other primary headaches. Electrodes are surgically implanted into brain structures, for example the posterior hypothalamus in case of cluster headache. Hypothalamic DBS has been successfully used to treat patients with cluster headache and short-lasting unilateral neuralgiform headache with conjunctival injection and tearing (SUNCT). In these headache conditions, DBS may reduce headache frequency by half, however, some side effects have also been reported that are mainly related to infection where the electrodes are placed, syncope, and diplopia. Hypothalamic DBS is proposed to modulate neuronal structures that are directly or indirectly involved in detection or transmission of painful stimuli or in the processing of this information. Reports of the use of DBS for chronic migraine are not found in the literature. This commentary explores the medical hypothesis that DBS could be considered as a therapeutic option for intractable chronic migraine. This hypothesis is based on the commonalities
\end{abstract}

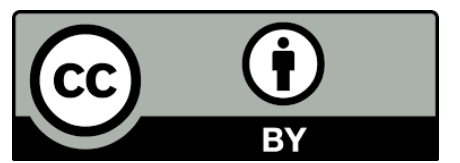

(C) 2021 by the author. This is an open access article distributed under the conditions of the Creative Commons by Attribution License, which permits unrestricted use, distribution, and reproduction in any medium or format, provided the original work is correctly cited. 
between cluster headache and migraine in terms of some shared symptoms, and potential overlap of underlying mechanisms. However, due to the absence of clinical evidence supporting the use of DBS in CM, the fact that disease-specific optimization of DBS parameters must be considered carefully, and considering the optimal CNS structures to target are currently unknown, it is concluded that this interventional and invasive technique should be avoided for $\mathrm{CM}$ for the present time.

\section{Keywords}

Deep brain stimulation; migraine; cluster headache; hypothalamus; headache

\section{Medical Hypothesis}

Chronic migraine $(\mathrm{CM})$ is a debilitating neurological disorder. Here, we put forward the hypothesis that deep brain stimulation (DBS) is a potential late option to treat severe intractable cases of $\mathrm{CM}$ and that the benefit is greater than the risks for those affected patients. This hypothesis has been formed based on 1) the observation that increased hypothalamic activity is associated with both cluster headache ( $\mathrm{CH}$ ) [1] and migraine [2] attacks; and 2) the demonstration that electrical stimulation of the hypothalamus (hypothalamic DBS) is effective for treating $\mathrm{CH}$ [3-5]. These observations support the idea that DBS might be beneficial for CM; however, the nature of this technique is invasive and side effects [6] may occur. Therefore, to test this hypothesis, appropriate $\mathrm{CM}$ patient selection must be taken into consideration. It is proposed that DBS be limited to use in the most complicated cases, where no other option is available, such as resistance to all other forms of CM therapy $[4,7]$.

\section{Chronic Migraine}

The International Headache Society classification of headache disorders (ICHD-3) defines CM as "headache occurring on $\geq 15$ days/month for more than 3 months with features of migraine on $\geq 8$ days/month" [8]. Up to $5 \%$ of the general population are affected by CM [9]. CM is less common than episodic migraine (EM); however, headache-related disability is higher and consequently the quality of life of affected individuals is lower [10-12]. Annually, about 3\% of patients with EM progress to CM [13]. Similar symptoms of migraine may accompany CM that include nausea, vomiting, photophobia, and phonophobia, but these are often less pronounced compared with EM [14]. Co-morbid conditions such as psychiatric and other somatic medical conditions are also higher in CM [15], leaving the affected individuals with a complex, difficult-to-treat condition. The burden of CM [16] is considerable and poses both individual and societal consequences $[17,18]$.

It is not yet clear how or for what reason EM progresses into CM in some individuals [19]; however, some potentially modifiable risk factors have been identified, which include the frequency of headache attacks, medication overuse, insufficient acute treatment, stress, and obesity [13, 20]. Identification of risk factors and application of appropriate treatments for acute migraine attacks can reduce headache frequency, severity, and disability; however, patients with CM are generally considered candidates for prophylactic therapy [21, 22]. Pharmacological options are limited, but 
high-quality evidence (level A) exists for the preventive therapy of CM with FDA approved agents such as propranolol, divalproex sodium, botulinum neurotoxin A, and topiramate [23, 24]. Nevertheless, a systematic review, including both observational studies and data from randomized clinical trials [25] has highlighted that poor patient adherence exists for migraine prophylaxis regimens that can be due to low tolerability or frequent dosing intervals. In addition, many prophylactic agents are only partially effective; providing $50 \%$ relief in approximately $50 \%$ of patients [26], while the side effects and cost of prolonged treatment often limit patient compliance. A recent meta-analysis [27] indicates that the newly introduced CGRP monoclonal antibodies (eptinezumab, erenumab, galcanezumab, and fremanezumab) are effective in CM, but long-term safety is still under investigation [28]. Alternative techniques such as behavioral and cognitive therapy [29], exercise, and stress management [23] have also been used with variable beneficial outcomes [30]. Those patients with CM who use available pharmacological treatments and still have inadequate pain relief or disabling side effects may turn to other therapeutic modalities, such as neuromodulation.

\section{Neuromodulation in Migraine}

A number of neuromodulatory strategies has been introduced for prevention of CM [31], which include non-invasive peripheral neurostimulation techniques such as occipital nerve stimulation, transcutaneous supraorbital stimulation, transcranial direct current stimulation, and repetitive magnetic stimulation [32]. These techniques need to be investigated further and are often among the later therapeutic choices for complex cases of CM resistant to other types of treatments [33]. It is beyond the scope of this manuscript to give a complete systematic review of all neurostimulation techniques investigated for migraine. We refer the readers to excellent reviews already available [7, 34-38].

The basis for neuromodulation in $\mathrm{CM}$ stems from evidence that demonstrates that migraine is a disorder of neuroplasticity and that migraine chronification results from a maladaptive process, which leads to the development of a hyper-excitability state in the brain. Several hypotheses exist to explain the pathogenesis of $\mathrm{CM}$, such as functional brain changes in affected patients, including increased cortical excitability, central trigemino-thalamic sensitization, and dysfunctional descending pain modulation [39-41]. These alterations have been linked to comorbid conditions, including analgesic overuse, chronic stress, anxiety, or depression that can further influence painprocessing pathways in migraine [40]. Sensitization of trigeminal and thalamic neurons has been postulated to be a potential key mechanism in the progression from EM to $\mathrm{CM}[40,42]$. The reversal of such plastic changes in the brain has led researchers to become interested in applying brain stimulation as a potential therapeutic option for migraine [32]. These techniques induce changes of neural plasticity that persist beyond the period of stimulation, which is fundamental to the prophylactic treatment of migraine, as they may target the neurophysiological abnormalities that contribute to the transition from EM to CM [32]. CEFALY ${ }^{\odot}$ is a commercially available device that stimulates the supraorbital branch of the trigeminal nerve with electrodes placed on the forehead. For the prevention of EM, a 20 min stimulation once a day with the CEFALY device was demonstrated to decrease headache days per month compared with the sham stimulation group ( $p$ $=0.054$ ) [43]. In 2019, Chou et al. [44] showed that treatment with CEFALY ${ }^{\odot}$ could abort pain during 
acute migraine attacks. However, the efficacy of this device for prophylaxis of CM is not yet supported by sufficient evidence. The technique is safe and easy to use $[45,46]$.

Vagus nerve stimulation (VNS) with an implanted vagus nerve stimulator was originally used for epileptic patients with migraine comorbidity, where it was found that it could improve migraine in these patients and therefore was considered as an option for migraine [47]. GammaCore ${ }^{\circledR}$ is a medical device developed to provide non-invasive VNS. This device is applied to the skin over the cervical branch of the vagus nerve and its use in animal models has been found to reduce cortical spreading depression [48]. In 2014, Oshinsky et al. [49] presented data on VNS that indicated it could reduce allodynia and that this effect was correlated with levels of extracellular glutamate in the subnucleus caudalis $(\mathrm{Vc})$ of the spinal trigeminal nucleus. Non-invasive VNS has been demonstrated to reduce pain during migraine attacks within 20 min of headache onset [50]. However, in the EVENT study by Silberstein et al. [51], use of non-invasive VNS in CM patients was not beneficial. This technique is also safe, but substantial evidence for its beneficial effects in CM is lacking.

Remote electrical neuromodulation (REN) has also been used to abort acute pain during migraine attacks $[52,53]$. In this technique, peripheral nerves of the upper arm are stimulated through a wireless wearable battery-operated stimulation unit controlled by a smartphone software application. The device is worn on the lateral upper arm to stimulate small cutaneous $C$ and $A \delta$ fibers for $45 \mathrm{~min}$ [54]. According to the manufacturer, the stimulation energy is low enough to maintain the overall sensory experience below perceptual pain threshold, at so called "subnociceptive" intensity. In contrast to transcutaneous electrical nerve stimulation (TENS) that is sensed as touch because it excites primarily $A \beta$ fibers, and produces pain relief through inhibition of ascending pain (gate control theory), Nerivio $^{\mathrm{TM}}$ induces pain relief by activation of brain stemmediated descending pain inhibition. This phenomenon is known as conditioned pain modulation (CPM), where a second painful stimulus can reduce the original pain [55]. Producers of $\mathrm{Nerivio}^{\mathrm{TM}}$ have also reported that stimulation by this device triggers global release of serotonin and norepinephrine, hence reinforcing endogenous analgesic mechanisms. Current data [54] show that Nerivio $^{\mathrm{TM}}$ has a favorable safety profile for the acute treatment of migraine.

Transcranial direct current stimulation (tDCS) is another technique that works by modulating cerebral cortical excitability and has been postulated to reduce migraine, since cortical spreading depression (CSD) [56] is a possible trigger of migraine [57]. A systematic review of 8 studies on tDCS in the prevention of migraine has provided evidence for a positive effect [58]. However, due to the small sample size and large degree of heterogeneity in the studies included in this systematic review, the quality of evidence is low and needs further confirmatory studies.

The other available techniques are transcranial magnetic stimulation (TMS) and repetitive TMS (rTMS) that are also proposed to modulate CSD [57, 59]. Experimental data in rats and cats have shown that single pulse TMS could block experimentally induced CSD [60]. Andreou et al. [60] elegantly presented data that CSD can be blocked in vivo by single pulse TMS and highlighted its thalamocortical modulatory capacity. This study was the first to show that the most important region of action was on thalamic neurons and that the trigeminocervical complex appeared less involved [60]. A systematic review by Stilling et al. in 2019 [58], which included both rTMS and TMS studies, presented data for an overall beneficial outcome in terms of reduction in headache frequency, duration, intensity, and analgesic use for acute migraine pain. Spring $\mathrm{TMS}^{\odot}$, a portable and rechargeable medical device was tested in a trial in 2010 [61], where it was demonstrated that $39 \%$ of patients became pain-free at $2 \mathrm{~h}$ after application of the device on the occipital region and 
the administration of two stimulations [61]. Two studies from 2015 [62] and 2018 [63] also reported efficacy and tolerability of the device in migraineurs with or without aura. Adverse effects related to the application of this device have been dizziness, tinnitus, and worsening of migraine, but with mild to moderate intensity and transient features [62,63].

In addition to the above mentioned non-invasive techniques, there are invasive techniques to treat primary headaches [38]. Transient stimulation of the spheno-palatine ganglion (SPG), chronic occipital nerve stimulation (ONS), and stimulation of retro-hypothalamic area by DBS are currently listed as invasive stimulation techniques [38]. Spheno-palatine ganglion stimulation (SPGS) has been proposed to work through several mechanisms, one of which is targeting the parasympathetic nervous system involved in the pathophysiology of trigeminal autonomic cephalalgias [64]. Pulsante ${ }^{\circledR}$ is a medical device, developed for SPGS to abort $\mathrm{CH}$ attacks in patients with chronic $\mathrm{CH}$ (CCH). SPGS might also be indicated in patients with $\mathrm{CM}$, but evidence is lacking in this population [38].

Although a number of studies [34,65] have provided evidence for somewhat beneficial effects of ONS, for example a significant reduction in headache days and disability [66], the exact underlying mechanism is still debated. It is proposed that ONS probably works by modulation of the trigeminocervical complex [67]. However, a delayed response in many patients has opened up the possibility of a more complex mechanism that involves non-specific regulation of central pain control systems. Functional neuroimaging techniques have provided evidence of metabolic changes following ONS in the regions suggested as pain processing regions, in particular in the anterior cingulate cortex [68].

DBS is the therapeutic use of chronic electrical stimulation of the brain via an implanted electrode [69]. It is most commonly used to treat the motor symptoms of Parkinson's disease (PD), and has occasionally been used or investigated for the treatment of a wide variety of other neurological and psychiatric conditions [70] including epilepsy, obsessive-compulsive disorder (OCD), major depression, and $\mathrm{CCH}$. DBS in the posterior hypothalamus is the most commonly used and most promising method for reduction of pain in refractory $\mathrm{CCH}$. Other brain targets that include stimulation at the junction between the diencephalic and mesencephalic tegmentum [71-73] have also been suggested and tried for $\mathrm{CCH}$. Readers are referred to papers on the effectiveness of DBS to treat attacks of $\mathrm{CH}$ assessed by Leone's group [3-5, 74, 75] and others [73, 76].

DBS is considered a last choice option for prevention of $\mathrm{CH}$ in patients with $\mathrm{CCH}$ who fulfill the selection criteria, namely at least 2 years of disease duration, at least one attack per day, resistance to pharmacotherapy, headache limited to the same side, normal neurological examination, and absence of psychiatric comorbidity [4, 7].

\section{DBS for $\mathrm{CCH}$}

DBS was originally proposed for $\mathrm{CCH}$ to target the attack generator, which was hypothesized to be located in the posterior hypothalamic region [74, 75]. This hypothesis was supported by functional neuroimaging techniques that demonstrated that this region is activated during $\mathrm{CH}$ attacks [1, 77]. Long-term hypothalamic stimulation was first reported in 2001 in a patient with $\mathrm{CCH}$ [78], where pain relief was observed with no major side effects. This technique was then further used for 16 drug-resistance $\mathrm{CCH}$ patients [79]. Long-term follow up after 4 years showed that 10 patients remained in a pain-free state [79]. 
According to Pedersen et al. [80], in addition to an inhibitory effect of DBS on the $\mathrm{CH}$ generator, located in the hypothalamus or the adjacent mesencephalic gray, two other potential mechanisms might explain the DBS effect in $\mathrm{CH}$. One theory describes activation of the periaqueductal gray (PAG) and/or rostral ventromedial medulla (RVM), that are both parts of the pain modulatory system, as the mechanism by which DBS decreases pain. The second theory describes a potential long-term effect on the pain-processing pathways $[81,82]$. The exact targeted structures that lead to a beneficial effect of DBS for $\mathrm{CCH}$ are still debated. However, collectively, it has been proposed that effective DBS for $\mathrm{CCH}$ modulates a more widespread network of the brain, rather than a single region, and the network potentially includes the trigeminal nuclei, PAG, hypothalamus, thalamus and cortical areas [83]. Advancement in neuroimaging studies, including PET and resting-state fMRI, shed further light into the contribution of the hypothalamus/midbrain tegmentum in $\mathrm{CH}$, and consequently different targets for DBS were considered such as inferior-posterior hypothalamus, midbrain tegmentum, and ventral tegmental area (for review see [84]). Further investigation also found changes in functional connectivity of not only these regions, but also relevant connections to pain-related areas, supporting the hypothesis that a complex matrix might be involved in modulating $\mathrm{CH}$ attacks [84].

Recently, Nowacki and his team have published a meta-analysis [85] to present estimates and predictors of long-term pain relief based on the cohorts of patients undergoing DBS for $\mathrm{CCH}$. This group found that in 40 patients in four different cohorts, mean reduction in headache frequency was $77 \%$ during a follow-up of 44 months, with an overall response rate of $75 \%$. A map of brain region stimulation for therapeutic effect of DBS identified the midbrain ventral and retrorubral tegmentum as important sites [85]. This meta-analysis also reinforces the current view that DBS in the ventral tegmental area probably acts upon pain circuits involved in maintaining $\mathrm{CH}$ [36]. Due to the finding that the therapeutic effect takes several weeks to manifest, it has been hypothesized that DBS in the ventral tegmentum induces a functional modulation of the pain processing network rather than pure inhibition of hypothalamic activity $[77,86]$.

Interestingly, trigeminal perception and pain thresholds have been measured following posterior hypothalamic DBS and reports showed that pain perception is only moderately decreased in peripheral limbs [87]. However, electrical pain thresholds or nociceptive reflexes within the trigeminal innervation territory remained unchanged [87]. Another study [88] has demonstrated that, in patients with drug-resistant $\mathrm{CCH}$ undergoing posterior hypothalamic DBS, cold pain thresholds in the receptive field of the ophthalmic branch of the trigeminal nerve were increased on the DBS stimulated side. The DBS group also had higher cold detection thresholds compared to non-implanted cluster headache patients. The authors of this study concluded that neurostimulation of the posterior hypothalamus is specific for $\mathrm{CH}$ and only certain aspects of pain sensation can be affected [88]. Collectively, these observations also point to a more complex mechanism of action underlying hypothalamic DBS for $\mathrm{CCH}$, rather than a direct, simple analgesic effect.

Only a proportion of patients respond to the DBS procedure. The reason is not completely understood. It was originally proposed that placement of electrode might be a potential reason. However, findings from a PET study [86] demonstrated that failure of DBS for CCH is not directly related to electrode placement, which suggests that other factors might be involved. No clinical or physiological marker can be used to assess the correct positioning of the electrodes. In addition, it seems that identification of exact stimulated areas is challenging. In an MRI study [89], which 
investigated the effect of stimulating electrode locations in patients with drug-resistant $\mathrm{CCH}$, no difference was found in anatomical location of stimulating electrodes between responders and nonresponders.

Nevertheless, DBS is not a risk-free procedure, is not considered a routine treatment for $\mathrm{CH}$, and is only used in centers with extensive experience. Although the number of cases with critical safety issues, such as hemorrhage, is limited, risk of bleeding is around 3\% [34]. Several cases of panic attack with dysautonomia, oculomotor disturbances, intraoperative transient ischemic attack, subcutaneous infection, transient loss of consciousness with hemiparesis and micturition syncope, erectile dysfunction, headache and tremor, euphoria, and paroxysmal sneezing have been reported in the literature [34]. On the other hand, sleep quality has been shown improved following posterior hypothalamic DBS in $\mathrm{CH}$ by the suppression of nocturnal attacks [90].

\section{DBS for CM}

According to the results of our literature search, clinical use of DBS for CM has not been reported. This might be due to the fact that several noninvasive and somewhat effective neuromodulatory techniques are available for migraine [31]. However, none of the currently available pharmacological or non-pharmacological strategies produces $100 \%$ efficacy and hence one can hypothesize that other options could be effective for those who are not responsive to the currently available treatments. One such option may be DBS. Since DBS has shown promise for treatment of patients with debilitating refractory $\mathrm{CCH}$ who are not responsive to other available treatments, it is hypothesized that patients with intractable CM might benefit from DBS. This medical hypothesis is based on evidence that shows that migraine and $\mathrm{CH}$ [91] share common features, which include certain clinical signs and symptoms, overlap with activated brain regions, and response to both pharmacological and neuromodulatory treatments. These observations point to a common link between these two types of primary headaches that most likely stems from underlying pathophysiological mechanisms, for example hyperexcitability of the brain network, progressive changes in nociceptive thresholds, and subsequent central sensitization [92]. Successful targeting of both conditions with triptans, some neurostimulation techniques, and novel data on CGRP monoclonal antibodies also suggest that both conditions may share some overlapping pathways [93, 94]. For a comprehensive review of the links between migraine and $\mathrm{CH}$, please see [95]. Figure 1 depicts some of the common features between $\mathrm{CH}$ and $\mathrm{CM}$. 

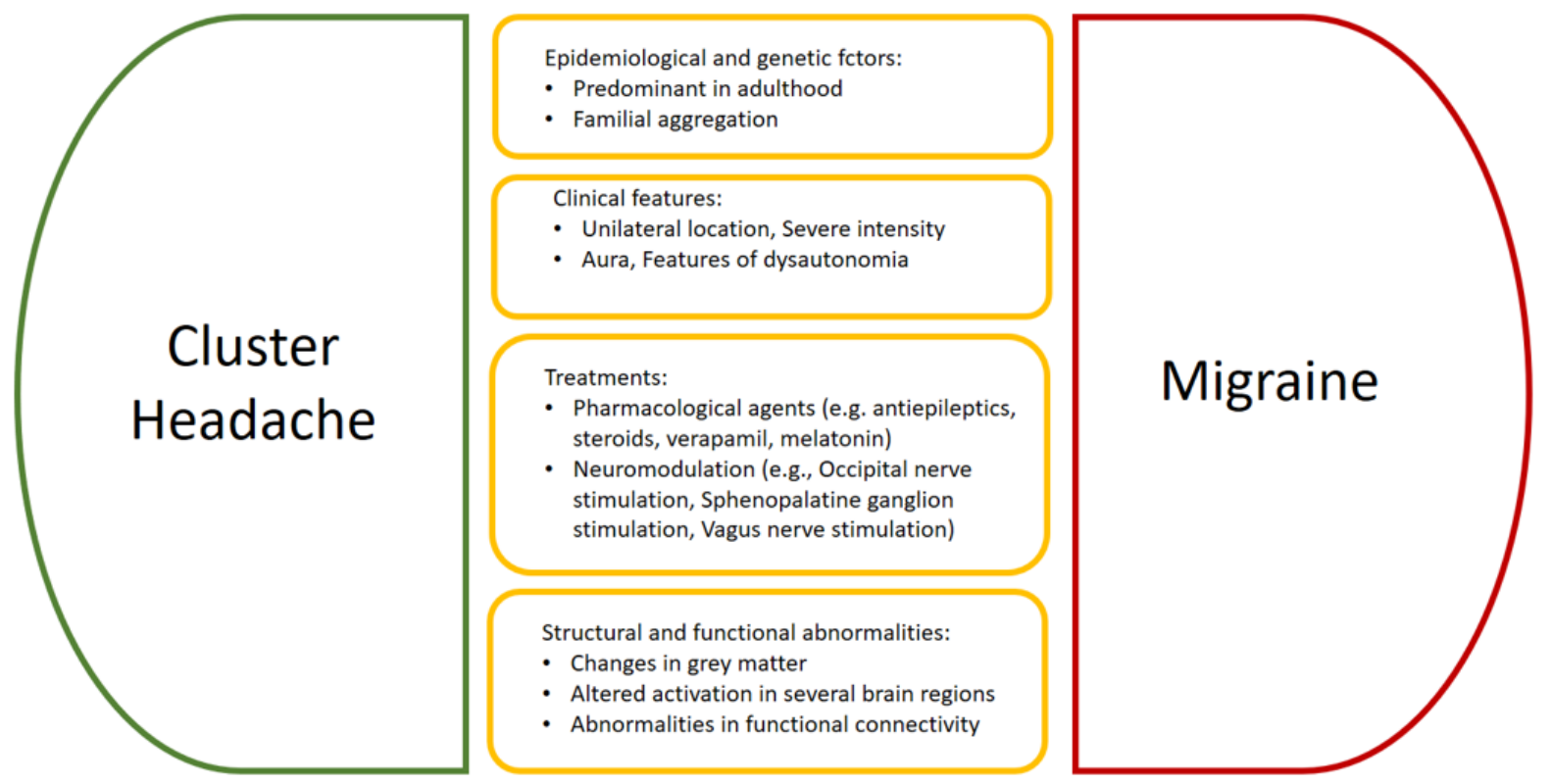

Figure 1 Examples of common features between cluster headache and migraine. Several characteristics share commonalities between these two primary headaches from clinical aspects to brain images, and response to some treatment strategies. For a comprehensive review, please see [92].

One interesting observation is the hyperperfusion, as a surrogate parameter for increased brain activity in the hypothalamus, that occurs both in $\mathrm{CH}$ and in migraine attacks [95]. Maniyar et al. [96] have found that activity in the hypothalamus is increased in the early premonitory phase of migraine, supporting a migraine mediator role for hypothalamus in addition to activation in the brain stem, the dorsal rostral pons, and the PAG [42, 96, 97]. Leone's group firstly hypothesized that highfrequency hypothalamic stimulation would inhibit apparent hyperactivity of this brain area and his theory was tested successfully in 2001 [78]. Progress on a putative mechanism of action of hypothalamic stimulation benefitted from PET studies in $\mathrm{CH}$ patients treated with hypothalamic DBS. In these patients, hypothalamic stimulation provoked increased blood flow in both the ipsilateral posterior inferior hypothalamic grey at the site of the electrode tip, and the ipsilateral trigeminal system [86], where for the first time a functional connection between the hypothalamus and the trigeminal system in humans was documented in vivo. Interestingly, activation of the trigeminal system did not provoke any headache attack, trigeminal pain, or features of autonomic craniofacial stimulations, and these findings led to the hypothesis that the activation of trigeminal system might contribute to $\mathrm{CH}$ attack but on its own cannot explain the attacks [86]. This observation, together with required latency of chronic stimulation to see the clinical effects of DBS propose a complex mechanism that might include the modulation of the antinociceptive system, i.e., increased threshold for cold pain [88]. Additionally, hypothalamic stimulation has resulted in elevated blood flow in brain areas known as pain matrix, such as the thalamus, somatosensory cortex, precuneus, and anterior cingulate cortex, but decreased blood flow in the middle temporal gyrus, posterior cingulate cortex, and insula [86]. Collectively, the evidence shows that hypothalamic stimulation interferes with components of this matrix and its therapeutic role might be by a gradual restoration of the normal function and metabolism in hypometabolic brain areas in patients with $\mathrm{CCH}$ [98]. This 
interpretation [99] is based on the accumulated experience that has changed the view of the hypothalamus as a generator of the $\mathrm{CH}$ attack to a modulator of the $\mathrm{CH}$ attack [100].

$\mathrm{CH}$ is also associated with dysautonomias, which are a group of conditions in which changes in the activity or function of the autonomic nervous system (ANS) negatively affect health [101]. Thus, the effects of DBS on the function of ANS has also attracted increased interest [102]. In a study by Cortelli et al, in 2007 [6], in patients with $\mathrm{CH}$ who were receiving posterior hypothalamic stimulation to identify the effects on headache, the orthostatic sympathoexcitatory response showed an increase during the head uptilt testing (HUTT) [6]. When DBS was off, a 3\% fall was observed in systolic blood pressure during the HUTT. However, when DBS stimulation was on, systolic blood pressure was maintained when the HUTT was repeated. An increase was observed in both diastolic blood pressure and total peripheral resistance for HUTT during the on versus the off mode of DBS stimulation [6]. These findings suggested that DBS could affect the peripheral vascular system. Heart rate variability (HRV), an index of autonomic activity, is widely used in clinical studies. Vagal parasympathetic effects cause high-frequency changes in heart rate. Hypothalamic stimulation in patients with $\mathrm{CH}$ increases the ratio of low-frequency to high-frequency HRV components. This finding suggests that hypothalamic DBS could activate the sympathetic nervous system. In contrast, during the $\mathrm{CH}$ attack in untreated patients, a reverse HRV pattern was observed, where a significant reduction in low-frequency HRV values was found, that is interpreted as autonomic nervous system dysfunction [103]. Other cardiovascular abnormalities have also been reported in patients during the $\mathrm{CH}$ attacks, including increases or decreases in heart rate and rhythm, atrial fibrillation, and heart block $[103,104]$. Collectively, these data provide evidence that autonomic function is also altered by hypothalamic DBS. For a review, see [102].

As the exact mechanism underlying DBS efficacy in $\mathrm{CCH}$ remains uncertain [35, 37], a discussion of how DBS might work for CM remains purely speculative.

Theoretically, one can consider other mechanisms that might underlie hypothalamic DBS for CM. DBS has been found to increase extracephalic pain thresholds in some $\mathrm{CH}$ patients [35, 37]. This suggests that deep brain stimulation may be reducing output from the posterior hypothalamus. In rats, it has been shown that there are direct connections between the paraventricular nucleus (PVN) of the hypothalamus and the trigeminal $\mathrm{Vc}$, where trigeminovascular neurons that relay painful input from the dura are located [105]. Inhibition of the PVN decreases the response of these Vc neurons to meningeal stimulation, which suggests that this input from the PVN to the $V_{c}$ is excitatory and thus pronociceptive. One possibility is that DBS, by activating local inhibitory interneurons in the PVN, decreases output from the PVN to the Vc. However, this possibility is inconsistent with the PET study in $\mathrm{CH}$ discussed above, which indicated increased neuronal activity not only in the PVN but also in the Vc after long term DBS for $\mathrm{CH}$ [86]. One way to account for this finding in human $\mathrm{CH}$ patients is to propose that the use of DBS over time results in neuroplastic changes in the $\mathrm{V} c$. These changes would involve the formation of synaptic connections from the PVN to local inhibitory interneurons within the Vc. As a result, DBS of the PVN would, over time, increase the inhibitory tone in the $\mathrm{Vc}_{\mathrm{c}}$, and thus increase the activation threshold of trigeminovascular neurons. Figure 2 illustrates these two potential mechanisms.

Similar to $\mathrm{CH}$, dysautonomias also accompany migraine attacks [106]; however, it is too early to comment on any potential effects of DBS on dysautonomias in migraine. 


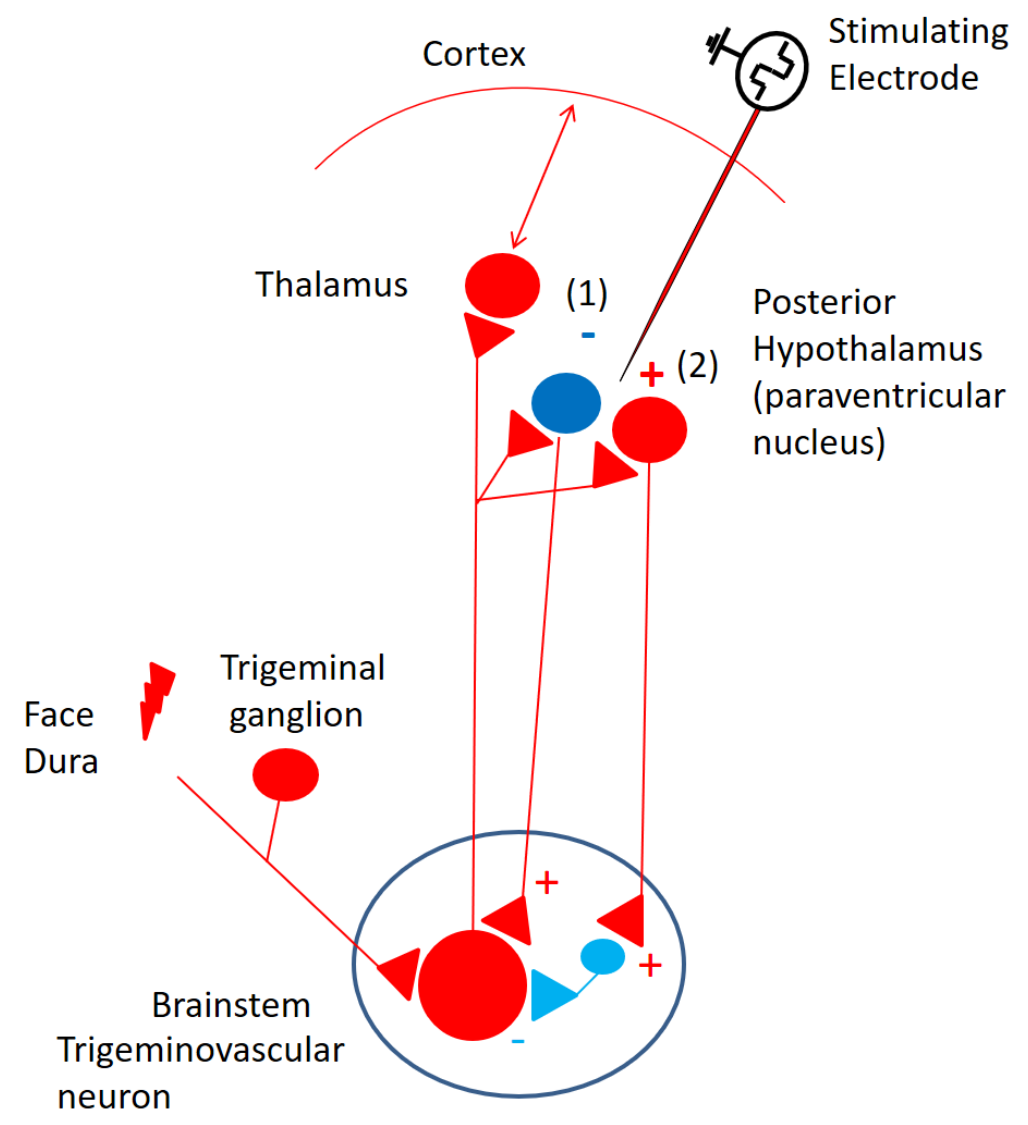

Figure $2 \mathrm{~A}$ theoretical model to present how DBS of the posterior hypothalamus could act to decrease CM. Red indicates excitation, blue inhibition. Nociceptive input from the dura and face activates second order trigeminovascular neurons, which transmit this information to the sensory cortex by way of the thalamus. The hypothalamus makes excitatory connections with the $\mathrm{Vc}$ that increase trigeminovascular neuron excitability. (1) DBS of the posterior hypothalamus over time decreases hypothalamic output, decreasing trigeminal sensory neuron excitability. (2) DBS of the posterior hypothalamus over time results in new connections to inhibitory interneurons in the $\mathrm{Vc}$, decreasing trigeminal sensory neuron excitability. Theoretically, either mechanism would lead to higher thresholds for headache pain, and/or decreased intensity of this pain.

\section{Safety and Patient Selection for DBS}

Transient visual disturbances, diplopia, vertigo, nausea, euphoria, bradycardia, and appetite changes have been reported as the main side effects following DBS of the posterior hypothalamus. Serious events have also been documented such as intracerebral hemorrhage, hardware infection, transient loss of consciousness, and skin erosion or hardware malfunction [107]. Lead fracture is another common complication. In a follow up study (1996-2007) [108], the long-term incidence of lead fractures in patients who underwent DBS surgery was determined. This retrospective study [108] was conducted on 208 patients who received 387 DBS electrodes, where 18 lead fractures were identified in 16 patients. Radiological screening has been suggested to determine the location and incidence of lead fracture in patients [108]. 
Considering the invasive nature of DBS, and associated risks, one must consider that use of DBS for CM, if it were to become an option, must be limited to a patient who is intolerant or has failed to achieve benefit from medications or non-invasive neuromodulation techniques. Currently, due to lack of sufficient evidence, it is not possible to determine the risk versus benefit for DBS in the treatment of CM. Hence, future potential use of DBS for CM should be considered based on each individual patient's case in relation to safety, and tolerability. Similar to the process of initiation of DBS for $\mathrm{CH}$, which started from individual cases, or case series, the use of DBS for CM would be considered on a case by case basis, and neurologists would be encouraged to report their findings if they have employed this technique for one or more CM patients. Lessons learned from small trials in $\mathrm{CH}$ [109] can be a useful starting point for CM. Fontaine et al [109] performed the first prospective crossover, double-blind, multicenter study assessing the efficacy and safety of unilateral hypothalamic DBS in 11 patients with severe refractory $\mathrm{CCH}$. Active and sham stimulation were compared during 1-month periods within the randomized part of the trial. Then, the study was followed by an open label phase for one year [109]. Researchers assessed headache severity and frequency, pain intensity, sumatriptan injection use, emotional impact, and quality of life. The randomized phase of this study did not support the efficacy of DBS in refractory $\mathrm{CCH}$, but findings from the open phase suggested long-term efficacy in more than $50 \%$ patients. Three serious adverse events were recorded, which included subcutaneous infection, transient loss of consciousness, and micturition syncope [109]. Comments raised about this trial by Ambrosini and Schoenen [110] highlight several points to be considered. Their concerns may also be applicable for a potential feasibility study for use of DBS in CM. Based on the findings from the randomized part of the study, Ambrosini and Schoenen [110] raised a valid point about the duration of treatment, which was that one month might be a rather short period for a therapeutic effect due to the potential for DBS to induce slow neuromodulatory changes. The delayed response in the open phase of the study shows that responders needed a longer duration of stimulation to benefit from DBS [110].

Patient selection with the aid of biomarkers, such as neuroimaging biomarkers [111], for example evidence of activation of hypothalamus [112], potential bio-based biomarkers, such as plasma levels of CGRP or certain miRNAs $[113,114]$, or predictive biomarkers for migraine chronification, such as polymorphism of certain genes [115], would allow for proper patient selection and stratification of patients (EM, and CM) prior to the DBS trials in CM.

It is important to note that $\mathrm{CM}$ is often complicated by other conditions, for example medication overuse headache [116], or can coexist with other disorders, such as epilepsy [117]. Therefore, it is also rational to consider, pre-DBS, if other treatments should be considered, for example a period of the detoxification therapy in the case of medication overuse headache [118]. In addition, for conditions comorbid with CM, it is logical to consider if potential use of DBS for those conditions may improve CM. For example, a case study [119] has shown that following anterior thalamic DBS for drug-resistant idiopathic generalized seizure, improvement has also been noticed in CM.

Collectively, the literature supports the notion of careful patient selection for DBS, i.e., restricted to patients who are resistant to all available treatments, including non-invasive preventive treatments. It also supports a longer time course of DBS treatment, in addition to a rational selection of primary and secondary end-points for determination of efficacy. Based on sufficient evidence, statements and guidelines could be developed similar to what is already available for DBS to treat $\mathrm{CCH}$ [7]. Such guidelines could also be drafted in the future, evaluated, and approved for CM. For an optimal DBS in CM, one must also consider that DBS for CM must be adjusted to be 
disease-specific. Therefore, the pattern of DBS application (i.e. parameters of frequencies and intensities) and format (i.e., continuous or intermittent) should be first optimized for CM. In addition, it is useful to report if any observation presents that co-existing conditions with CM undergoing DBS could indirectly affect migraine, because this would allow collecting relevant data and potential target structure for stimulation. Both positive and negative data must be reported. For example, an indirect beneficial effect in CM has been reported following anterior thalamic DBS for a case of coexisting epilepsy [119], however, subthalamic DBS in a case of Parkinson's disease [120], did not exert any significant change for CM co-existed with the Parkinson's disease (see the abstract P196 in [120]).

\section{Conclusion}

This commentary presents a medical hypothesis that the use of DBS might open a new therapeutic avenue for CM patients. Based on evidence that DBS has been effective in $\mathrm{CH}$ patients, and the fact that the pathogenesis of $\mathrm{CH}$ and $\mathrm{CM}$ shares some common features, we presented some pros and cons of considering DBS for CM. On one hand, this invasive technique could be justified in cases of $\mathrm{CM}$ who are not responders to any available treatments. A balance between benefit and harm needs to be carefully considered, based on the safety and long-term outcomes of DBS for $\mathrm{CH}$, prior to patient selection and, for that reason, DBS for CM would be expected to initially involve individual patient cases. Ethical considerations and safety issues must also be justified caseby case to minimize the risks to patients. A clear communication between patients and clinicians would help to identify potential benefits and risks, patient preference, clinician's recommendation, and available options prior to a final decision. Case reports from these individual patient trials might then be used to justify if further evaluation of DBS might be an option for randomized controlled or open label studies. The individual patient trial data would also suggest the length of treatment needed before significant improvement in CM symptoms might be expected. In addition, due to the fact DBS might be used for those conditions that co-exist with CM, observations of improvement in $\mathrm{CM}$, while treating such co-existing conditions must be reported. This would allow collection of relevant data and potentially the structural targets for beneficial DBS for CM. This approach has already appeared in the literature, where DBS for epilepsy [119] and Parkinson's disease [120], was reported to exert beneficial or no effects on $\mathrm{CM}$, respectively. Similarly, it was also reported that subthalamic DBS not only produced beneficial effects in advanced Parkinson's disease but could also alleviate $\mathrm{CH}$ [121].

In short, DBS could be of potential benefit for CM, either directly or indirectly, where CM coexists with other conditions that are being treated with DBS. The potential use of DBS for chronic, debilitating CM resistant to other non-invasive alternatives remains open, but at this stage, we await accumulation of sufficient clinical evidence before any general statement can be formulated for application of DBS in CM. In line with this view, disease-specific optimization of DBS parameters that include the most relevant CNS structures to be targeted with DBS for CM must be considered. Such parameters for $\mathrm{CM}$ remain to be determined. 


\section{Author Contributions}

PG came up with the article concept, conducted the literature review, and wrote the draft article. BEC contributed to the writing of the article and creation of the figures. PG and BEC contributed to manuscript revision, read and approved the submitted version.

\section{Competing Interests}

The authors have declared that no competing interests exist.

\section{References}

1. May A, Bahra A, Buchel C, Frackowiak RS, Goadsby PJ. Hypothalamic activation in cluster headache attacks. Lancet. 1998; 352: 275-278.

2. Denuelle M, Fabre N, Payoux P, Chollet F, Geraud G. Hypothalamic activation in spontaneous migraine attacks. Headache. 2007; 47: 1418-1426.

3. Leone M, Franzini A, Broggi G, May A, Bussone G. Long-term follow-up of bilateral hypothalamic stimulation for intractable cluster headache. Brain. 2004; 127: 2259-2264.

4. Leone M, May A, Franzini A, Broggi G, Dodick D, Rapoport A, et al. Deep brain stimulation for intractable chronic cluster headache: Proposals for patient selection. Cephalalgia. 2004; 24: 934-937.

5. Leone M, Proietti Cecchini A, Franzini A, Broggi G, Cortelli P, Montagna P, et al. Lessons from 8 years' experience of hypothalamic stimulation in cluster headache. Cephalalgia. 2008; 28: 787797.

6. Cortelli P, Guaraldi P, Leone M, Pierangeli G, Barletta G, Grimaldi D, et al. Effect of deep brain stimulation of the posterior hypothalamic area on the cardiovascular system in chronic cluster headache patients. Eur J Neurol. 2007; 14: 1008-1015.

7. Martelletti P, Jensen RH, Antal A, Arcioni R, Brighina F, de Tommaso M, et al. Neuromodulation of chronic headaches: Position statement from the European Headache Federation. J Headache Pain. 2013: 14; 86.

8. Headache classification committee of the international headache society (IHS) The international classification of headache disorders, 3rd edition. London: Cephalalgia; 2018.

9. Natoli JL, Manack A, Dean B, Butler Q, Turkel CC, Stovner L, et al. Global prevalence of chronic migraine: A systematic review. Cephalalgia. 2010; 30: 599-609.

10. Buse D, Manack A, Serrano D, Reed M, Varon S, Turkel C, et al. Headache impact of chronic and episodic migraine: Results from the American Migraine Prevalence and Prevention study. Headache. 2012; 52: 3-17.

11. Bigal ME, Rapoport AM, Lipton RB, Tepper SJ, Sheftell FD. Assessment of migraine disability using the migraine disability assessment (MIDAS) questionnaire: A comparison of chronic migraine with episodic migraine. Headache. 2003; 43: 336-342.

12. Meletiche DM, Lofland JH, Young WB. Quality-of-life differences between patients with episodic and transformed migraine. Headache. 2001; 41: 573-578.

13. Bigal ME, Serrano D, Buse D, Scher A, Stewart WF, Lipton RB. Acute migraine medications and evolution from episodic to chronic migraine: A longitudinal population-based study. Headache. 2008; 48: 1157-1168. 
14. Yalin OO, Uluduz D, Ozge A, Sungur MA, Selekler M, Siva A. Phenotypic features of chronic migraine. J Headache Pain. 2016; 17: 26.

15. Buse DC, Manack A, Serrano D, Turkel C, Lipton RB. Sociodemographic and comorbidity profiles of chronic migraine and episodic migraine sufferers. J Neurol Neurosurg Psychiatry. 2010; 81: 428-432.

16. Leonardi $M$, Raggi A. A narrative review on the burden of migraine: When the burden is the impact on people's life. J Headache Pain. 2019; 20: 41.

17. Lipton RB, Silberstein SD. Episodic and chronic migraine headache: Breaking down barriers to optimal treatment and prevention. Headache.2015; 55: 103-122.

18. Bigal ME, Serrano D, Reed M, Lipton RB. Chronic migraine in the population: Burden, diagnosis, and satisfaction with treatment. Neurology. 2008; 71: 559-566.

19. Katsarava Z, Buse DC, Manack AN, Lipton RB. Defining the differences between episodic migraine and chronic migraine. Curr Pain Headache Rep. 2012; 16: 86-92.

20. Bigal ME, Lipton RB. Modifiable risk factors for migraine progression. Headache. 2006; 46: 13341343.

21. Sun-Edelstein C, Rapoport AM. Update on the pharmacological treatment of chronic migraine. Curr Pain Headache Rep. 2016; 20: 6.

22. Agostoni EC, Barbanti P, Calabresi P, Colombo B, Cortelli P, Frediani F, et al. Current and emerging evidence-based treatment options in chronic migraine: $A$ narrative review. J Headache Pain. 2019; 20: 92.

23. May A, Schulte LH. Chronic migraine: Risk factors, mechanisms and treatment. Nat Rev Neurol. 2016; 12: 455-464.

24. Peters GL. Migraine overview and summary of current and emerging treatment options. Am J Manag Care. 2019; 25: S23-S34.

25. Hepp Z, Bloudek LM, Varon SF. Systematic review of migraine prophylaxis adherence and persistence. J Manag Care Pharm. 2014; 20: 22-33.

26. Shukla R, Sinh M. Migraine: Prophylactic treatment. J Assoc Physicians India. 2010; 58 : 26-29.

27. Han L, Liu $Y$, Xiong $H$, Hong P. CGRP monoclonal antibody for preventive treatment of chronic migraine: An update of meta-analysis. Brain Behav. 2019; 9: e01215.

28. Deen M, Correnti E, Kamm K, Kelderman T, Papetti L, Rubio-Beltrán E, et al. Blocking CGRP in migraine patients-a review of pros and cons. J Headache Pain. 2017; 18: 96.

29. Harris P, Loveman E, Clegg A, Easton S, Berry N. Systematic review of cognitive behavioural therapy for the management of headaches and migraines in adults. Br J Pain. 2015; 9: 213-224.

30. Karakurum Goksel B. The use of complementary and alternative medicine in patients with migraine. Noro Psikiyatr Ars. 2013; 50: S41-S46.

31. Puledda F, Shields K. Non-pharmacological approaches for migraine. Neurotherapeutics. 2018; 15: 336-345.

32. Schoenen J, Roberta B, Magis D, Coppola G. Noninvasive neurostimulation methods for migraine therapy: The available evidence. Cephalalgia. 2016; 36: 1170-1180.

33. Vigano A, Toscano M, Puledda F, Di Piero V. Treating chronic migraine with neuromodulation: The role of neurophysiological abnormalities and maladaptive plasticity. Front Pharmacol. 2019; 10: 32.

34. Magis D, Schoenen J. Advances and challenges in neurostimulation for headaches. Lancet Neurol. 2012; 11: 708-719. 
35. Schwedt TJ, Vargas B. Neurostimulation for Treatment of Migraine and Cluster Headache. Pain Med. 2015; 16: 1827-1834.

36. Miller S, Sinclair AJ, Davies B, Matharu M. Neurostimulation in the treatment of primary headaches. Pract Neurol. 2016; 16: 362-375.

37. Schwedt TJ. Neurostimulation for primary headache disorders. Curr Neurol Neurosci Rep. 2009; 9: 101-107.

38. Moisset $X$, Lanteri-Minet M, Fontaine D. Neurostimulation methods in the treatment of chronic pain. J Neural Transm (Vienna). 2020; 127: 673-686.

39. Mathew NT. Pathophysiology of chronic migraine and mode of action of preventive medications. Headache. 2011; 51: 84-92.

40. Su M, Yu S. Chronic migraine: A process of dysmodulation and sensitization. Mol Pain. 2018; 14 : 1744806918767697.

41. Diener HC, Dodick DW, Goadsby PJ, Lipton RB, Olesen J, Silberstein SD. Chronic migraineclassification, characteristics and treatment. Nat Rev Neurol. 2012; 8: 162-171.

42. Goadsby PJ, Holland PR, Martins-Oliveira M, Hoffmann J, Schankin C, Akerman S. Pathophysiology of migraine: A disorder of sensory processing. Physiol Rev. 2017; 97: 553-622.

43. Meng F, Zhang J, Schoenen J, Vandersmissen B, Herroelen L, Vandenheede M, et al. Migraine prevention with a supraorbital transcutaneous stimulator: A randomized controlled trial. Neurology. 2013; 80: 697-704.

44. Chou DE, Shnayderman Yugrakh M, Winegarner D, Rowe V, Kuruvilla D, Schoenen J. Acute migraine therapy with external trigeminal neurostimulation (ACME): A randomized controlled trial. Cephalalgia. 2019; 39: 3-14.

45. Piquet M, Balestra C, Sava SL, Schoenen JE. Supraorbital transcutaneous neurostimulation has sedative effects in healthy subjects. BMC Neurol. 2011; 11: 135.

46. Magis D, D'Ostilio K, Thibaut A, De Pasqua V, Gerard P, Hustinx R, et al. Cerebral metabolism before and after external trigeminal nerve stimulation in episodic migraine. Cephalalgia. 2017 37; 881-891.

47. Lenaerts ME, Oommen KJ, Couch JR, Skaggs V. Can vagus nerve stimulation help migraine? Cephalalgia. 2008; 28: 392-395.

48. Chen SP, Ay I, de Morais AL, Qin T, Zheng Y, Sadeghian H, et al. Vagus nerve stimulation inhibits cortical spreading depression. Pain. 2016; 157: 797-805.

49. Oshinsky ML, Murphy AL, Hekierski Jr H, Cooper M, Simon BJ. Noninvasive vagus nerve stimulation as treatment for trigeminal allodynia. Pain. 2014; 155: 1037-1042.

50. Tassorelli C, Grazzi L, de Tommaso M et al. Noninvasive vagus nerve stimulation as acute therapy for migraine: The randomized PRESTO study. Neurology. 2018; 91: e364-e373.

51. Silberstein SD, Calhoun AH, Lipton RB, Grosberg BM, Cady RK, Dorlas S, et al. Chronic migraine headache prevention with noninvasive vagus nerve stimulation: The EVENT study. Neurology. 2016; 87: 529-538.

52. Yarnitsky D, Dodick DW, Grosberg BM, Burstein R, Ironi A, Harris D, et al. Remote electrical neuromodulation (REN) relieves acute migraine: A randomized, double-blind, placebocontrolled, multicenter trial. Headache. 2019; 59: 1240-1252.

53. Yarnitsky D, Volokh L, Ironi A, Weller B, Shor M, Shifrin A, et al. Nonpainful remote electrical stimulation alleviates episodic migraine pain. Neurology. 2017; 88: 1250-1255. 
54. Rapoport AM, Bonner JH, Lin T, Harris D, Gruper $\mathrm{Y}$, Ironi A, et al. Remote electrical neuromodulation (REN) in the acute treatment of migraine: $A$ comparison with usual care and acute migraine medications. J Headache Pain. 2019; 20: 83.

55. Yarnitsky D. Conditioned pain modulation (the diffuse noxious inhibitory control-like effect): Its relevance for acute and chronic pain states. Curr Opin Anaesthesiol. 2010; 23: 611-615.

56. Charles A, Brennan K. Cortical spreading depression-new insights and persistent questions. Cephalalgia. 2009; 29: 1115-1124.

57. Ferrari MD, Klever RR, Terwindt GM, Ayata C, van den Maagdenberg AM. Migraine pathophysiology: Lessons from mouse models and human genetics. Lancet Neurol. 2015; 14: 65-80.

58. Stilling JM, Monchi O, Amoozegar F, Debert CT. Transcranial magnetic and direct current stimulation (TMS/tDCS) for the treatment of headache: A systematic review. Headache. 2019; 59: 339-357.

59. Lloyd JO, Chisholm KI, Oehle B, Jones MG, Okine BN, Al-Kaisy A, et al. Cortical mechanisms of single-pulse transcranial magnetic stimulation in migraine. Neurotherapeutics. 2020.

60. Andreou AP, Holland PR, Akerman S, Summ O, Fredrick J, Goadsby PJ. Transcranial magnetic stimulation and potential cortical and trigeminothalamic mechanisms in migraine. Brain. 2016; 139: 2002-2014.

61. Lipton RB, Dodick DW, Silberstein SD, Saper JR, Aurora SK, Pearlman SH, et al. Single-pulse transcranial magnetic stimulation for acute treatment of migraine with aura: A randomised, double-blind, parallel-group, sham-controlled trial. Lancet Neurol. 2010; 9: 373-380.

62. Bhola R, Kinsella E, Giffin N, Lipscombe S, Ahmed F, Weatherall M, et al. Single-pulse transcranial magnetic stimulation (sTMS) for the acute treatment of migraine: Evaluation of outcome data for the UK post market pilot program. J Headache Pain.2015; 16: 535.

63. Starling AJ, Tepper SJ, Marmura MJ, Shamim EA, Robbins MS, Hindiyeh N, et al. A multicenter, prospective, single arm, open label, observational study of sTMS for migraine prevention (ESPOUSE Study). Cephalalgia. 2018; 38: 1038-1048.

64. Goadsby PJ. Pathophysiology of cluster headache: A trigeminal autonomic cephalgia. Lancet Neurol. 2002; 1: 251-257.

65. Saper JR, Dodick DW, Silberstein SD, McCarville S, Sun M, Goadsby PJ, et al. Occipital nerve stimulation for the treatment of intractable chronic migraine headache: ONSTIM feasibility study. Cephalalgia. 2011; 31: 271-285.

66. Dodick DW, Silberstein SD, Reed KL, Deer TR, Slavin KV, Huh B, et al. Safety and efficacy of peripheral nerve stimulation of the occipital nerves for the management of chronic migraine: Long-term results from a randomized, multicenter, double-blinded, controlled study. Cephalalgia. 2015; 35: 344-358.

67. Goadsby PJ, Bartsch T, Dodick DW. Occipital nerve stimulation for headache: Mechanisms and efficacy. Headache. 2008; 48: 313-318.

68. Magis D, Bruno MA, Fumal A, Gérardy PY, Hustinx R, Laureys $S$, et al. Central modulation in cluster headache patients treated with occipital nerve stimulation: An FDG-PET study. BMC Neurol. 2011; 11: 25.

69. Herrington TM, Cheng JJ, Eskandar EN. Mechanisms of deep brain stimulation. J Neurophysiol. 2016; 115: 19-38. 
70. Jakobs M, Fomenko A, Lozano AM, Kiening KL. Cellular, molecular, and clinical mechanisms of action of deep brain stimulation-a systematic review on established indications and outlook on future developments. EMBO Mol Med. 2019; 11: e9575.

71. Akram H, Miller S, Lagrata S, Hyam J, Jahanshahi M, Hariz M, et al. Ventral tegmental area deep brain stimulation for refractory chronic cluster headache. Neurology. 2016; 86: 1676-1682.

72. Chabardes S, Carron R, Seigneuret E, Torres N, Goetz L, Krainik A, et al. Endoventricular deep brain stimulation of the third Ventricle: Proof of concept and application to cluster headache. Neurosurgery. 2016; 79: 806-815.

73. Seijo-Fernandez F, Saiz A, Santamarta E, Nader L, Alvarez-Vega MA, Lozano B, et al. Long-term results of deep brain stimulation of the mamillotegmental fasciculus in chronic cluster headache. Stereotact Funct Neurosurg. 2018; 96: 215-222.

74. Leone M. Deep brain stimulation in headache. Lancet Neurol. 2006; 5: 873-877.

75. Leone M, Proietti Cecchini A. Deep brain stimulation in headache. Cephalalgia. 2016; 36: 11431148.

76. Akram H, Miller S, Lagrata S, Hariz M, Ashburner J, Behrens T, et al. Optimal deep brain stimulation site and target connectivity for chronic cluster headache. Neurology. 2017; 89: 2083-2091.

77. May A, Ashburner J, Buchel C, McGonigle DJ, Friston KJ, Frackowiak RS, et al. Correlation between structural and functional changes in brain in an idiopathic headache syndrome. Nat Med. 1999; 5: 836-838.

78. Leone M, Franzini A, Bussone G. Stereotactic stimulation of posterior hypothalamic gray matter in a patient with intractable cluster headache. N Engl J Med. 2001; 345: 1428-1429.

79. Leone M, Franzini A, Broggi G, Bussone G. Hypothalamic stimulation for intractable cluster headache: long-term experience. Neurology. 2006; 67: 150-152.

80. Pedersen JL, Barloese $M$, Jensen RH. Neurostimulation in cluster headache: A review of current progress. Cephalalgia. 2013; 33: 1179-1193.

81. May A. Hypothalamic deep-brain stimulation: Target and potential mechanism for the treatment of cluster headache. Cephalalgia. 2008; 28: 799-803.

82. Bussone G, Franzini A, Proietti Cecchini A, Mea E, Curone M, Tullo V, et al. Deep brain stimulation in craniofacial pain: Seven years' experience. Neurol Sci. 2007; 28: S146-149.

83. Nowacki A, Moir L, Owen SL, Fitzgerald JJ, Green AL, Aziz TZ. Deep brain stimulation of chronic cluster headaches: Posterior hypothalamus, ventral tegmentum and beyond. Cephalalgia. 2019; 39: 1111-1120.

84. Ferraro S, Nigri A, Demichelis G, Pinardi C, Chiapparini L, Giani L, et al. Understanding cluster headache using magnetic resonance imaging. Front Neurol. 2020; 11: 535.

85. Nowacki A, Schober M, Nader L, Saryyeva A, Nguyen TK, Green AL, et al. Deep brain stimulation for chronic cluster headache: Meta-analysis of individual patient data. Ann Neurol. 2020; 88: 956-969.

86. May A, Leone $M$, Boecker $H$, Sprenger $T$, Juergens $T$, Bussone $G$, et al. Hypothalamic deep brain stimulation in positron emission tomography. J Neurosci. 2006; 26: 3589-3593.

87. Schoenen J, Di Clemente L, Vandenheede M, Fumal A, De Pasqua V, Mouchamps M, et al. Hypothalamic stimulation in chronic cluster headache: A pilot study of efficacy and mode of action. Brain. 2005; 128: 940-945. 
88. Jurgens TP, Leone M, Proietti-Cecchini A, Busch V, Mea E, Bussone G, et al. Hypothalamic deepbrain stimulation modulates thermal sensitivity and pain thresholds in cluster headache. Pain. 2009; 146: 84-90.

89. Fontaine $D$, Lanteri-Minet $M$, Ouchchane L, Lazorthes $Y$, Mertens $P$, Blond $S$, et al. Anatomical location of effective deep brain stimulation electrodes in chronic cluster headache. Brain. 2010; 133: 1214-1223.

90. Vetrugno R, Pierangeli G, Leone M, Bussone G, Franzini A, Brogli G, et al. Effect on sleep of posterior hypothalamus stimulation in cluster headache. Headache. 2007; 47: 1085-1090.

91. Hoffmann J, May A. Diagnosis, pathophysiology, and management of cluster headache. Lancet Neurol. 2018; 17: 75-83.

92. Vollesen AL, Benemei S, Cortese F. Migraine and cluster headache-the common link. J Headache Pain. 2018; 19: 89.

93. Carmine Belin A, Ran C, Edvinsson L. Calcitonin Gene-Related Peptide (CGRP) and cluster headache. Brain Sci. 2020; 10: 30.

94. Chan C, Goadsby PJ. CGRP pathway monoclonal antibodies for cluster headache. Expert Opin Biol Ther. 2020; 20: 947-953.

95. Hansen JM, Schankin CJ. Cerebral hemodynamics in the different phases of migraine and cluster headache. J Cereb Blood Flow Metab. 2019; 39: 595-609.

96. Maniyar FH, Sprenger T, Monteith T, Schankin C, Goadsby PJ. Brain activations in the premonitory phase of nitroglycerin-triggered migraine attacks. Brain. 2014; 137: 232-241.

97. Bahra A, Matharu MS, Buchel C, Frackowiak RS, Goadsby PJ. Brainstem activation specific to migraine headache. Lancet. 2001; 357: 1016-1017.

98. Sprenger T, Ruether KV, Boecker H, Valet M, Berthele A, Pfaffenrath V, et al. Altered metabolism in frontal brain circuits in cluster headache. Cephalalgia. 2007; 27: 1033-1042.

99. Leone M, Franzini A, Cecchini AP, Broggi G, Bussone G. Hypothalamic deep brain stimulation in the treatment of chronic cluster headache. Ther Adv Neurol Disord. 2010; 3: 187-195.

100. Leone M, Bussone G. Pathophysiology of trigeminal autonomic cephalalgias. Lancet Neurol. 2009; 8: 755-764.

101.Goldstein DS, Robertson D, Esler M, Straus SE, Eisenhofer G. Dysautonomias: Clinical disorders of the autonomic nervous system. Ann Intern Med. 2002; 137: 753-763.

102. Hyam JA, Kringelbach ML, Silburn PA, Aziz TZ, Green AL. The autonomic effects of deep brain stimulation--a therapeutic opportunity. Nat Rev Neurol. 2012; 8: 391-400.

103. Tubani L, Baratta L, Giorgino F, Delfino M, Fiore G, Golluscio V, et al. Heart rate variability in cluster headache. Ann Ital Med Int. 2003; 18: 42-46.

104. Russell D, Storstein L. Cluster headache: A computerized analysis of $24 \mathrm{~h}$ Holter ECG recordings and description of ECG rhythm disturbances. Cephalalgia. 1983; 3: 83-107.

105. Robert C, Bourgeais L, Arreto CD, Condes-Lara M, Noseda R, Jay T, et al. Paraventricular hypothalamic regulation of trigeminovascular mechanisms involved in headaches. J Neurosci. 2013; 33: 8827-8840.

106. Gazerani P, Cairns BE. Dysautonomia in the pathogenesis of migraine. Expert Rev Neurother. 2018; 18: 153-165.

107. Vyas DB, Ho AL, Dadey DY, Pendharkar AV, Sussman ES, Cowan R, et al. Deep brain stimulation for chronic cluster headache: A review. Neuromodulation. 2019; 22: 388-397. 
108. Fernandez FS, Alvarez Vega MA, Antuna Ramos A, Fernandez Gonzalez F, Lozano Aragoneses B. Lead fractures in deep brain stimulation during long-term follow-up. Parkinsons Dis. 2010; 2010: 409356.

109. Fontaine D, Lazorthes $Y$, Mertens P, Blond S, Géraud G, Fabre N, et al. Safety and efficacy of deep brain stimulation in refractory cluster headache: $A$ randomized placebo-controlled double-blind trial followed by a 1-year open extension. J Headache Pain. 2010; 11: 23-31.

110. Ambrosini A, Schoenen J. Commentary on Fontaine et al.: "Safety and efficacy of deep brain stimulation in refractory cluster headache: A randomized placebo-controlled double-blind trial followed by a 1-year open extension". J Headache Pain. 2010; 11: 21-22.

111. Russo A, Silvestro $M$, Tessitore A, Tedeschi G. Functional neuroimaging biomarkers in migraine: Diagnostic, prognostic and therapeutic implications. Curr Med Chem. 2019; 26: 6236-6252.

112. Peres MF, Sanchez del Rio M, Seabra ML, Tufik S, Abucham J, Cipolla-Neto J, et al. Hypothalamic involvement in chronic migraine. J Neurol Neurosurg Psychiatry. 2001; 71: 747-751.

113. Greco R, De Icco R, Demartini C, Zanaboni AM, Tumelero E, Sances G, et al. Plasma levels of CGRP and expression of specific microRNAs in blood cells of episodic and chronic migraine subjects: Towards the identification of a panel of peripheral biomarkers of migraine? J Headache Pain. 2020; 21: 122.

114.Gazerani P. Current evidence on potential uses of microrna biomarkers for migraine: From diagnosis to treatment. Mol Diagn Ther. 2019; 23: 681-694.

115. Yakubova A, Davidyuk Y, Tohka J, Khayrutdinova O, Kudryavtsev I, Nurkhametova D, et al. Searching for predictors of migraine chronification: A pilot study of $1911 \mathrm{~A}>\mathrm{G}$ polymorphism of TRPV1 gene in episodic versus chronic migraine. J Mol Neurosci. 2020.

116. Vandenbussche N, Laterza D, Lisicki M, Lloyd J, Lupi C, Tischler H, et al. Medication-overuse headache: A widely recognized entity amidst ongoing debate. J Headache Pain. 2018; 19: 50.

117. Verrotti A, Striano P, Belcastro V, Matricardi S, Villa MP, Parisi P. Migralepsy and related conditions: Advances in pathophysiology and classification. Seizure. 2011; 20: 271-275.

118. Kristoffersen ES, Grande RB, Aaseth K, Russell MB, Lundqvist C. Medication-overuse headache detoxification reduces headache disability-the Akershus study of chronic headache. Eur J Neurol. 2018; 25: 1140-1147.

119. Lendvai IS, Kinfe TM. Migraine improvement after anterior thalamic deep brain stimulation for drug-resistant idiopathic generalized seizure: A case report. Headache. 2017; 57: 964-966.

120.11th European headache federation congress jointly with 31st congress of the Italian society for the study of headaches. J Headache Pain. 2017; 18: 111.

121. Huotarinen A, Reich M, Volkmann J, Pekkonen E. STN DBS for advanced parkinson disease simultaneously alleviates cluster headache. Case Rep Neurol. 2017; 9: 289-292. 


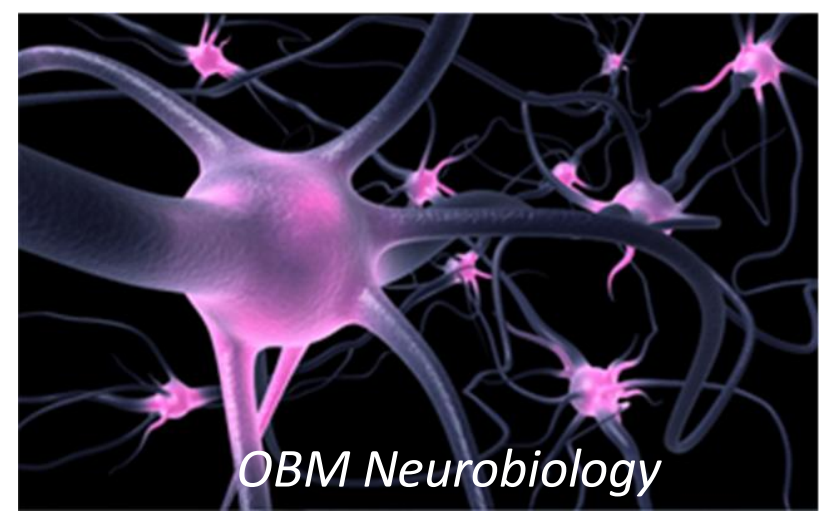

Enjoy OBM Neurobiology by:

1. Submitting a manuscript

2. Joining volunteer reviewer bank

3. Joining Editorial Board

4. Guest editing a special issue

For more details, please visit:

http://www.lidsen.com/journals/neurobiology 